\title{
Polarizability tensor and Kramers-Heisenberg induction
}

\author{
C. M. J. Wijers \\ Faculty of Applied Science, University of Twente, P. O. Box 217, 7500 AE Enschede, The Netherlands
}

(Received 8 July 2004; published 13 December 2004)

\begin{abstract}
A general expression for the semiclassical, nonrelativistic linear polarizability of an arbitrary volume element $V$ has been derived in the long wavelength approximation. The derivation starts from the expectation value of the dipole strength, as in the original Kramers-Heisenberg paper about optical scattering by atoms. The main requirements underlying the present approach are a separate non-Hermitian part of the Hamiltonian and a frequency dependent damping, which is zero for the static case. Resonant and antiresonant exponentials are both found to be necessary to obtain a proper static response. It is concluded that even parity for the damping has to be preferred from the theoretical point of view, although odd and asymmetric parity yield virtually the same polarizability. The electromagnetic response can still be written in terms of a single complex frequency, in agreement with the requirements of electrodynamics. The resulting expression is suited for the treatment of nonisotropic systems.
\end{abstract}

DOI: 10.1103/PhysRevA.70.063807

PACS number(s): 42.65.An, 32.10.Dk, 33.15.Kr

\section{INTRODUCTION}

The basic and fundamental problem of the optical response of atoms to an externally applied electric field was treated by Kramers and Heisenberg in a classical paper [1], as early as 1925 . This treatment was improved and extended by Dirac [2] and subsequently by Weiskopf and Wigner [3]. The backbone of the Kramers-Heisenberg (KH) paper consists of the classical Larmor expression for the total intensity scattered by the dipole strength induced in the atom by the applied field and the calculation of this dipole strength by what is called now first order linear time dependent perturbation theory. The surprising aspect of the paper is that, although definitely the induced dipole strength depends in a linear fashion upon the amplitude of the electric field, the paper does not even discuss why this dependency has not been used to define properly a corresponding polarizability. This deficiency prevents the induction studied in the paper, from being applied in an easy and direct way to problems of electrodynamics. A number of later papers presented polarizability expressions classified as Kramers-Heisenberg, but despite the, at first glance, simple character of the derivation, there is still no consensus about the precise formulation, as can be inferred from recent publications [4-6]. This holds particularly for the frequency dependent parity of the damping. To investigate this parity issue, the general expression for the semiclassical nonrelativistic polarizability of an arbitrary volume element $V$ of subwavelength dimensions will be (re)derived in this paper, as in the original $\mathrm{KH}$ paper, starting from the dipole strength. A separate, dissipative, time and frequency dependent part of the Hamiltonian in the single electron picture will be used for the quantum mechanical part of the derivation. This is equivalent to the use of complex self-energies in methods of condensed matter physics, like the GW or Bethe-Salpeter equations methods [7]. This dissipative part will determine directly the damping of the polarizability, but only as far as material energy losses are concerned. The energy stored in the electromagnetic field is not part of the Hamiltonian. As a result the radiative selfdamping cannot follow from this description [8-10].
The ongoing discussion about the subject finds its origin in the requirement from electromagnetism that the time dependence has to be described by means of a real valued cosine. As a result, two complex exponentials, resonant and antiresonant, are necessary to link quantum mechanics and electrodynamics. The asymmetric treatment in quantum mechanics of the resonant and antiresonant exponential prevents a smooth connection from being made, since electromagnetism requires a symmetric treatment. Therefore, in this paper the two exponentials necessary in the quantum mechanical description will be contracted into a single one complying with the electrodynamic demands. As only external electromagnetic fields (KH: "Der Einfluß äußerer Bestrahlung") are taken into account, a finite volume $V$ is implied. As a result, electrodynamic potentials can be used, without having to cope with gauge transform problems. The definition of the polarizability itself requires that $V$ has to be small enough for a long wavelength approximation to be allowed. In the original KH paper only isotropic types of response were treated. This restriction is not necessary and some elementary tensor rules [11] suffice to extend the treatment to arbitrary anisotropic cases.

Along these lines, an expression for the polarizability of an arbitrary volume element will be derived, which can be used for quantitative descriptions and is suited to handle arbitrary polarization dependent behavior. This expression will be highly useful for problems where the emphasis is on the material aspect of the light-matter interaction and copes with the frequency dependent parity aspects of the corresponding damping.

\section{TIME DEPENDENT PERTURBATION THEORY}

The classical derivation of the Kramers-Heisenberg expression is based upon first order linear perturbation theory. Accordingly, the present treatment will start from the assumption that the system is described by the time dependent Schrödinger equation 


$$
\begin{gathered}
{\left[H_{0}+\hat{H}_{D}(\omega)+W(\mathbf{r}) f(t)\right] \Psi(\mathbf{r}, t)=i \hbar \frac{\partial}{\partial t} \Psi(\mathbf{r}, t),} \\
f(t)=e^{i \omega t},
\end{gathered}
$$

where $H_{0}$ is the Hermitean ground state Hamiltonian of the material system. The time dependent perturbation $W(\mathbf{r}) f(t)$ stems from sources external to the system and will be described at the start by a single complex exponential of positive frequency $\omega . \hat{H}_{D}(\omega)$ is the dissipative non-Hermitian part of the Hamiltonian, and is zero for $\omega=0[3,8]$, the case of a time independent perturbation. In this paper, the caret will be used to indicate that a quantity is complex. $\hat{H}_{D}(\omega)$ can also be identified as the interaction of the electron with the bath or reservoir in the effective one-electron description [12]. For the ground state it is assumed that the complete and orthonormal set of eigensolutions $E_{k}^{(0)}, \phi_{k}^{(0)}(\mathbf{r})$ in the independent particle treatment of the problem, is known. For the perturbed Hamiltonian $H_{0}+\hat{H}_{D}(\omega)$ a different set of orthonormal solutions $\hat{E}_{k}^{(1)}, \phi_{k}^{(1)}(\mathbf{r})$ has to be taken. This set has the same solutions for the occupied states, but has slightly different ones for unoccupied or excited states, the more pronounced difference being the nonzero imaginary part of the (quasi)energies. For these states it will be used that

$$
\left\langle\phi_{l}^{(1)}\left|H_{0}+\hat{H}_{D}(\omega)\right| \phi_{l}^{(1)}\right\rangle=\hat{E}_{l}^{(1)}=E_{l}^{(1)}-i \hbar \gamma(\omega) .
$$

Since $\hat{H}_{D}$ is a function of $\omega, \gamma$ should also be a function of frequency, $\gamma(\omega)$.

To start, only a single pair of levels, involving an occupied state $k$ and an unoccupied state $l$, will be considered, for which the following energy differences will be used:

$$
\begin{gathered}
E_{l k}=E_{l}^{(0)}-E_{k}^{(0)}=\hbar \omega_{l k}, \\
\hat{E}_{l k}=\hat{E}_{l}^{(1)}-E_{k}^{(1)} \approx E_{l}^{(0)}-E_{k}^{(0)}-i \hbar \gamma_{l k}(\omega) \\
=\hbar \hat{\omega}_{l k}=\hbar\left[\omega_{l k}-i \gamma_{l k}(\omega)\right] .
\end{gathered}
$$

The damping $\gamma$ depends upon the indices $l k$. This dependence, however, will not be shown in the derivation to keep the expressions more transparent. The corresponding ansatz for the wave function, as perturbed by $W(\mathbf{r}) f(t)$, is in the pair approximation:

$$
\Psi(\mathbf{r}, t)=c_{k}(t) \phi_{k}^{(1)}(\mathbf{r}) e^{-i E_{k}^{(1)} t / \hbar}+c_{l}(t) \phi_{l}^{(1)}(\mathbf{r}) e^{-i \hat{E}_{l}^{(1)} t / \hbar} .
$$

The damping $\gamma(\omega)$, contained here in $\hat{E}_{l}^{(1)}$, has to be positive, because of the dissipative character of the perturbed Hamiltonian $H_{0}+\hat{H}_{D}(\omega)$. To denote the damping of the probability/ intensity $\Gamma$ is mostly used, related to $\gamma$ by $\Gamma=2 \gamma$. By means of ansatz (4), the Schrödinger equation can be solved up to first order with the set $\hat{E}_{k}^{(1)}, \phi_{k}^{(1)}(\mathbf{r})$ being a solution of $H_{0}$ $+\hat{H}_{D}(\omega)$ :

$$
\begin{gathered}
c_{k}(t) W(\mathbf{r}) f(t) \phi_{k}^{(1)}(\mathbf{r}) e^{-i E_{k}^{(1)} t / \hbar}+c_{l}(t) W(\mathbf{r}) f(t) \phi_{l}^{(1)}(\mathbf{r}) e^{-i \hat{E}_{l}^{(1)} t / \hbar} \\
=i \hbar \phi_{k}^{(1)}(\mathbf{r}) e^{-i E_{k}^{(1)} t / \hbar} \frac{d}{d t} c_{k}(t)+i \hbar \phi_{l}^{(1)}(\mathbf{r}) e^{-i \hat{E}_{l}^{(1)} t / \hbar} \frac{d}{d t} c_{l}(t) .
\end{gathered}
$$

This result is multiplied by $\phi_{l}^{(1)^{*}}(\mathbf{r})$ and is next integrated over all space. For that step it is easiest to assume that the levels of the pair $l, k$ are nondegenerate. Degenerate levels can only be treated however, if they are properly orthogonalized (e.g., by Schmidt's procedure). If so, the orthonormality of the set $\phi_{l}^{(1)}$ can be used to obtain

$$
\begin{aligned}
i \hbar e^{-i \hat{E}_{l}^{(1)} t / \hbar} \frac{d}{d t} c_{l}(t)= & c_{k}(t) f(t) e^{-i E_{k}^{(1)} t / \hbar}\langle l|W(\mathbf{r})| k\rangle \\
& +c_{l}(t) f(t) e^{-i \hat{E}_{l}^{(1)} t / \hbar}\langle l|W(\mathbf{r})| l\rangle .
\end{aligned}
$$

The implicit assumption made in the $\mathrm{KH}$ derivation is the linearity with respect to the perturbation $W(\mathbf{r})$. In this paper only electric dipole type of perturbations will be considered, as treated in more detail in the next section. In Eq. (17) it will be shown that the external perturbation $W$ scales with the momentum operator $\mathbf{p}$ and the external vector potential $\mathbf{A}_{X}$. This vector potential will be assumed to be constant over that part of space where the wave function has most of its probability. Then the spatial parity of the perturbation $W(\mathbf{r})$ is the same as the spatial parity of the momentum operator $\mathbf{p}$ : antisymmetric. If the states in $V$ have also a defined spatial parity, it then holds that

$$
\int d \mathbf{r} \phi_{l}^{(1)^{*}}(\mathbf{r}) W(\mathbf{r}) \phi_{l}^{(1)}(\mathbf{r})=0
$$

This leaves only one term in the right-hand side of Eq. (6). Now one time integration step suffices to obtain the expression for the coefficient $c_{l}(t)$ of the excited state:

$$
\begin{aligned}
c_{l}(t) & =\frac{1}{i \hbar} \int_{0}^{t} d t^{\prime} e^{i\left(\hat{\omega}_{l k}+\omega\right) t^{\prime}}\langle l|W(\mathbf{r})| k\rangle \\
& =-\frac{\langle l|W(\mathbf{r})| k\rangle_{V}}{\hbar}\left[\frac{e^{i\left(\hat{\omega}_{l k}+\omega\right) t}-1}{\hat{\omega}_{l k}+\omega}\right] .
\end{aligned}
$$

The integration to obtain the expectation value of the perturbation $\langle l|W| k\rangle$ can be limited to the volume $V$, if it is assumed that the part of the wave function outside $V$ can be neglected.

For an electromagnetic wave taken as a perturbation, $f(t)$ is given by

$$
f(t)=f_{+}(t)+f_{-}(t)=\frac{1}{2}\left[e^{i \omega t}+e^{-i \omega t}\right]=\cos (\omega t) .
$$

After the Schrödinger equation (1) has been solved for $f_{+}(t)$ and $f_{-}(t)$ separately, the two solutions $\Psi_{+}(\mathbf{r}, t), \Psi_{-}(\mathbf{r}, t)$ can be added (as in $\mathrm{KH}$ ). This approximation is valid if the condition 


$$
f_{+}(t) \Psi_{-}(\mathbf{r}, t)-f_{-}(t) \Psi_{+}(\mathbf{r}, t) \approx 0
$$

is satisfied. This restriction holds rigorously for $\omega=0$ and is irrelevant for $\omega$ at resonance. Therefore it is a good approximation. Beyond these restrictions, the use of the rotating wave approximation is required, but at the expense of a much longer and less transparent derivation. Since most of the discussion to come will focus upon the static behavior, this extension is superfluous.

The cos $\omega t$ description (9) determines integrally the damping part of the Hamiltonian $\hat{H}_{D}(\omega)$. Since the cosine is even in $\omega$, both $\hat{H}_{D}(\omega)$ and $\gamma(\omega)$ can only be even functions of $\omega$ obviously. Both quantities have to be 0 for $\omega=0$. Despite these restrictions on $\gamma(\omega)$ by quantum mechanics, the parity of $\gamma(\omega)$ with respect to $\omega$ will be left undefined, to allow for an open analysis of the frequency dependent parity issue.

\section{ELECTRIC DIPOLE OPERATOR AND EXPECTATION VALUE}

As stated in the Introduction, the dipole strength $\langle\mathbf{d}\rangle_{V}(t)$, the expectation value of the electric dipole operator $\mathbf{d}$ over the volume $V$, is the main interface between quantum mechanics and electrodynamics. To describe this electric dipole operator, the real space representation will be used (as in the $\mathrm{KH}$ paper):

$$
\mathbf{d}=q \mathbf{r}
$$

where $\mathbf{r}$ is the spatial coordinate and $q$ the charge of the electron. This electromagnetic source term enters the Maxwell equations as an expectation value $\langle\mathbf{d}\rangle_{V}(t)$, given by

$$
\begin{aligned}
\langle\mathbf{d}\rangle_{V}(t)= & \langle\Psi(\mathbf{r}, t)|\mathbf{d}| \Psi(\mathbf{r}, t)\rangle_{V} \\
= & \left|c_{k}(t)\right|^{2}\left\langle\phi_{k}^{(1)}(\mathbf{r})|\mathbf{d}| \phi_{k}^{(1)}(\mathbf{r})\right\rangle_{V} \\
& +\left|c_{l}(t)\right|^{2}\left\langle\phi_{l}^{(1)}(\mathbf{r})|\mathbf{d}| \phi_{l}^{(1)}(\mathbf{r})\right\rangle_{V} \\
& +c_{k}^{*}(t) c_{l}(t)\left\langle\phi_{k}^{(1)}(\mathbf{r})|\mathbf{d}| \phi_{l}^{(1)}(\mathbf{r})\right\rangle_{V} e^{i\left(\hat{E}_{k}^{(1)}-\hat{E}_{l}^{(1)}\right) t / \hbar} \\
& +c_{l}^{*}(t) c_{k}(t)\left\langle\phi_{l}^{(1)}(\mathbf{r})|\mathbf{d}| \phi_{k}^{(1)}(\mathbf{r})\right\rangle_{V} e^{i\left(\hat{E}_{l}^{(1) *}-\hat{E}_{k}^{(1)}\right) t / \hbar}
\end{aligned}
$$

The first two terms can be neglected, because they are either too weak or almost static $\left[\left|c_{l}(t)\right| \ll\left|c_{k}(t)\right|,\left|c_{k}(t)\right|^{2}+\left|c_{l}(t)\right|^{2}=1\right]$. So, the approximate result is given by

$$
\begin{aligned}
\langle\mathbf{d}\rangle_{V}(t) & \approx 2 \operatorname{Re}\left[c_{l}(t) e^{-i \hat{\omega}_{l k} t}\langle k|\mathbf{d}| l\rangle_{V}\right] \\
& =-\frac{2}{\hbar} \operatorname{Re}\left[\left(\frac{e^{i \omega t}}{\hat{\omega}_{l k}+\omega}\right)\langle k|\mathbf{d}| l\rangle_{V}\langle l|W(\mathbf{r})| k\rangle_{V}\right] .
\end{aligned}
$$

This expectation value describes the contribution to the dipole strength $\langle\mathbf{d}\rangle$ of the volume $V$, due to transitions from state $k$ to state $l$. In the above reasoning, one exponential containing $\hat{\omega}_{l k}$ has been omitted, since it is strongly damped and only the steady state response is needed. The further derivation depends entirely upon the general expression for the one-particle Hamiltonian $H$ [13]:
$H \Psi(\mathbf{r}, t)$

$$
=\left[\left(\frac{p^{2}}{2 m}+q \Phi(\mathbf{r})\right)-\frac{q}{2 m}\left(\mathbf{p}^{T} \mathbf{A}+\mathbf{A}^{T} \mathbf{p}\right)+\frac{q^{2}}{2 m} A^{2}\right] \Psi(\mathbf{r}, t),
$$

which will be used to specify $W(\mathbf{r})$. As has been stated in the Introduction, the radiation field as such is not part of the Hamiltonian, since the derivation is semiclassical. We have used the convention that a vector becomes transposed when it has a superscript $T$. If a transposed vector is followed by a second vector the notation implies the scalar product. If a vector with superscript $T$ has been preceded by a second vector the notation implies a direct product tensor. The notation is particularly useful $[11,14]$ when applied to triple products, because of the associativity:

$$
\mathbf{a}\left(\mathbf{b}^{T} \mathbf{c}\right)=\left(\mathbf{a b}^{T}\right) \mathbf{c},
$$

where $\mathbf{a}, \mathbf{b}, \mathbf{c}$ are arbitrary vectors. The vector potential $\mathbf{A}(\mathbf{r}, t)$ will be decomposed into the following components:

$$
\mathbf{A}(\mathbf{r}, t)=\mathbf{A}_{0}(\mathbf{r})+\mathbf{A}_{I}(\mathbf{r}, t)+\mathbf{A}_{X}(\mathbf{r}, t),
$$

with all static components of $\mathbf{A}(\mathbf{r}, t)$ belonging to $\mathbf{A}_{0}(\mathbf{r})$. The dynamic part of $\mathbf{A}(\mathbf{r}, t)$ is attributed to $\mathbf{A}_{I}(\mathbf{r}, t)$ or $\mathbf{A}_{X}(\mathbf{r}, t)$, depending on whether the electromagnetic sources causing them are inside volume $V$ or not. For this reason the vector potential part $\mathbf{A}_{X}(\mathbf{r}, t)$ obeys the vacuum Maxwell equations inside the volume $V$. In conventional optics the static component $\mathbf{A}_{0}(\mathbf{r})$ can be ignored. When the term containing $A^{2}$ is neglected in the usual way and expression (16) for $\mathbf{A}(\mathbf{r}, t)$ is substituted into Eq. (14), the different components of $H$, as defined in Eq. (1), can be identified, giving

$$
\begin{gathered}
H_{0}=\frac{p^{2}}{2 m}+q \Phi(\mathbf{r}), \\
\hat{H}_{D}(\omega)=-\frac{q}{2 m}\left[\mathbf{p}^{T} \mathbf{A}_{I}(\mathbf{r}, t)+\mathbf{A}_{I}^{T}(\mathbf{r}, t) \mathbf{p}\right], \\
W(\mathbf{r}) f(t)=-\frac{q}{2 m}\left[\mathbf{p}^{T} \mathbf{A}_{X}(\mathbf{r})+\mathbf{A}_{X}^{T}(\mathbf{r}) \mathbf{p}\right] f(t) .
\end{gathered}
$$

The ground state Hamiltonian $H_{0}$ and the dissipative part of the Hamiltonian $\hat{H}_{D}(\omega)$ should also contain exchangecorrelation contributions when the number of electrons contributing to the response of the volume element $V$ exceeds 1 . Such additions will not influence, however, the main findings of this paper. The polarizability is defined with respect to the electric field $\mathbf{E}$. Therefore the general relation between the electrodynamic potentials $\mathbf{A}, \Phi$ and the electric field $\mathbf{E}$ is needed:

$$
\mathbf{E}(\mathbf{r}, t)=-\frac{\partial}{\partial t} \mathbf{A}(\mathbf{r}, t)-\boldsymbol{\nabla} \Phi(\mathbf{r}, t)
$$

and this requires a closer examination of the use of gauge transforms for this case. It is a common assumption in quantum mechanics that the scalar potential $\Phi$ is time independent: 


$$
\frac{\partial}{\partial t} \Phi(\mathbf{r}, t)=0
$$

Actually this assumption is a special kind of electrodynamic gauge. It is the only gauge that will be used in this paper. Since the contribution of the scalar potential to the electric field is now a static one, it can be neglected in the analysis of the dynamics of the problem. Next, as in the original $\mathrm{KH}$ paper, only the external field components will be taken into account in $W(\mathbf{r})$ :

$$
\mathbf{E}_{X}(\mathbf{r}, t)=g(t) \mathbf{E}_{X}(\mathbf{r})=-\frac{\partial f(t)}{\partial t} \mathbf{A}_{X}(\mathbf{r}) .
$$

From the entire Hamiltonian $H$ only the external perturbation $W(\mathbf{r})$ is needed to treat the electromagnetic source term $\langle\mathbf{d}\rangle$. To give this perturbation the commonly used shape, requires that the divergence of the external vector potential has to vanish. Since this external electric field $\mathbf{E}_{X}$ obeys the vacuum Maxwell equations inside the volume $V$ by definition, the Poisson equation can be applied, yielding

$$
\boldsymbol{\nabla}^{T} \mathbf{E}_{X}(\mathbf{r}, t)=-\frac{\partial f(t)}{\partial t} \boldsymbol{\nabla}^{T} \mathbf{A}_{X}(\mathbf{r})=0
$$

For all dynamic cases the time derivative $f^{\prime}(t)$ is nonzero. Hence the Poisson equation acts like the traditional Coulomb gauge here. Then rigorously the following operator equation holds:

$$
\boldsymbol{\nabla}^{T} \mathbf{A}_{X}=\boldsymbol{\nabla}^{T} \underline{A}_{X}+\mathbf{A}_{X}^{T} \boldsymbol{\nabla}=\mathbf{A}_{X}^{T} \boldsymbol{\nabla}
$$

The commutation of vector potential and gradient is necessary. Ignoring this, it would not be possible to define a polarizability using its standard definition. For a single atom or volume element $V$, the vector potential $\mathbf{A}_{X}$ is the external field. When more atoms or volume elements $V$ need to be taken into account, $\mathbf{A}_{X}$ is nothing else but the traditional local field. Often the field $\mathbf{A}_{X}$ is also called the applied field, but in that sense it mostly comprises both cases. The assumption commonly made in this kind of derivations is that the applied field $\mathbf{A}_{X}$ is constant over the volume $V$, the so-called long wavelength approximation. Also this assumption makes the divergence disappear, but that is not needed at this place. The perturbation can now be written as

$$
W(\mathbf{r}) f(t)=-\frac{q}{m} \mathbf{A}_{X}(\mathbf{r})^{T} \mathbf{p} f(t)=-\frac{q \hbar}{m i} \mathbf{A}_{X}(\mathbf{r})^{T} \boldsymbol{\nabla} f(t) .
$$

The subscript $X$ can be omitted, since the only fields going to be used will be the applied ones $\mathbf{A}(\mathbf{r})=\mathbf{A}_{X}(\mathbf{r})$. The commutator of $H_{0}$ and $\mathbf{r}$, according to Ehrenfest's theorem, can be taken from textbooks [13]. The vector potential $\mathbf{A}(\mathbf{r})$ can be replaced by the electric field $\mathbf{E}(\mathbf{r})$, using Eq. (18) and the exponential time dependence from Eq. (1), giving

$$
\left[\mathbf{r}, H_{0}\right]=\frac{i \hbar}{m} \mathbf{p},
$$

$$
\mathbf{A}(\mathbf{r})=-\frac{1}{i \omega} \mathbf{E}(\mathbf{r})
$$

These two relations enable us to rewrite the matrix element of $W(\mathbf{r})$ in the form

$$
\begin{aligned}
\langle l|W(\mathbf{r})| k\rangle_{V} & =-\frac{q}{i \hbar}\left(E_{k}^{(0)}-E_{l}^{(0)}\right)\langle l|\mathbf{r}| k\rangle_{V}^{T} \mathbf{A}(\mathbf{r}) \\
& =q\left(\frac{\omega_{l k}}{\omega}\right)\langle l|\mathbf{r}| k\rangle_{V}^{T} \mathbf{E}(\mathbf{r}) .
\end{aligned}
$$

Since the commutator is related to the ground state Hamiltonian, the frequency $\omega_{l k}$ is a real quantity. It is at this stage that the additional prefactor containing the reciprocal of the frequency $\omega$ shows up, which is known to occur in derivations based upon electromagnetic potentials [15]. The KH paper uses an $\mathbf{E}^{T} \mathbf{r}$-type of perturbation from the beginning, but in this paper the $\mathbf{A}^{T} \mathbf{p}$-type will be preferred to treat the optical response problem. If the classical relation $\mathbf{p}=m \dot{\mathbf{r}}$ were used, the prefactor would be just 1 , as at resonance, for $\omega=\omega_{l k}$. So the origin of the prefactor is a quantum mechanical one. At this stage the long wavelength approximation is used to bring $\mathbf{A}(\mathbf{r})$ outside the integral and the coordinate $\mathbf{r}$ now only points to the center of volume element $V$.

\section{POLARIZABILITY TENSOR}

The introduction of the polarizability tensor requires the amplitude of the electric field to be specified. This tensor is not an operator in the sense of quantum mechanics, unlike the dipole operator. The first specification of the electric field amplitude uses a single complex exponential of positive frequency for its time dependence and is directly connected to the quantum mechanical treatment of the preceding two sections:

$$
\mathbf{E}(\mathbf{r}, t)=\mathbf{E}(\mathbf{r}) e^{i \omega t}
$$

The derived matrix element $\langle l|W(\mathbf{r})| k\rangle_{V}$ can now be substituted into the expression for the dipole strength (13) to obtain the single frequency spectral component contributed by the pair $l k$ :

$$
\begin{gathered}
\langle\mathbf{d}\rangle_{V}(t)=\operatorname{Re}\left[\overleftrightarrow{\alpha}_{F}(\omega) \mathbf{E}(\mathbf{r}) e^{i \omega t}\right] \\
\stackrel{\leftrightarrow}{\alpha}_{F}(\omega)=-\frac{2 q^{2}}{\hbar}\left(\frac{\omega_{l k}}{\omega}\right)\left(\frac{\langle k|\mathbf{r}| l\rangle_{V}\langle l|\mathbf{r}| k\rangle_{V}^{T}}{\omega_{l k}+\omega-i \gamma(\omega)}\right)
\end{gathered}
$$

This polarizability $\alpha_{F}(\omega)$ represents a single frequency polarizability. Electromagnetism, however, requires a real valued electric field to be applied to the system. To satisfy that condition, a cosine description will be used of the form

$$
\mathbf{E}(\mathbf{r}, t)=\mathbf{E}(\mathbf{r}) \cos \omega t=\frac{1}{2}\left[e^{i \omega t}+e^{-i \omega t}\right] \mathbf{E}(\mathbf{r}) .
$$

Although an arbitrary electric field is described by $\cos (\omega t$ $+\phi$ ), only a time translation suffices to reduce the description to the proposed one. The electric field (28) introduces an inseparable positive and negative frequency exponential, as 
discussed before, and, according to Eq. (27), gives rise to the following direct description of the dipole strength:

$$
\begin{aligned}
\langle\mathbf{d}\rangle_{V}(t)= & \frac{q^{2}}{\hbar}\left(\frac{\omega_{l k}}{\omega}\right) \operatorname{Re}\left[\left(\frac{e^{-i \omega t}}{\omega_{l k}-\omega-i \gamma(-\omega)}-\frac{e^{i \omega t}}{\omega_{l k}+\omega-i \gamma(\omega)}\right)\right. \\
& \left.\times\langle k|\mathbf{r}| l\rangle_{V}\langle l|\mathbf{r}| k\rangle_{V}^{T} \mathbf{E}(\mathbf{r})\right] .
\end{aligned}
$$

In this paper it will be assumed that the matrix elements $\langle k|\mathbf{r}| l\rangle_{V}$ will be real, as is the case in conventional optics. At first, the special resonant case of $\omega \approx \omega_{l k}$ will be considered, with only the first exponential inside the square brackets contributing:

$$
\begin{gathered}
\langle\mathbf{d}\rangle_{V}(t)=\operatorname{Re}\left[\mathbf{d}(-\omega) e^{-i \omega t}\right]=\operatorname{Re}\left[\overleftrightarrow{\alpha}_{R}(-\omega) \mathbf{E}(\mathbf{r}) e^{-i \omega t}\right], \\
\overleftrightarrow{\alpha}_{R}(\omega)=-\frac{q^{2}}{\hbar}\left(\frac{\omega_{l k}}{\omega}\right)\left[\frac{1}{\omega_{l k}+\omega-i \gamma(\omega)}\right]\langle k|\mathbf{r}| l\rangle_{V}\langle l|\mathbf{r}| k\rangle_{V}^{T}
\end{gathered}
$$

with $\omega$ the positive frequency of the cosine form of $\mathbf{E}$. This resonant polarizability allows the direct result (29) to be rewritten as

$$
\begin{aligned}
\langle\mathbf{d}\rangle_{V}(t) & =\operatorname{Re}\left[\mathbf{d}(-\omega) e^{-i \omega t}+\mathbf{d}(\omega) e^{i \omega t}\right] \\
& =\operatorname{Re}\left[\left(\overleftrightarrow{\alpha}_{R}(-\omega) e^{-i \omega t}+\overleftrightarrow{\alpha}_{R}(\omega) e^{i \omega t}\right) \mathbf{E}(\mathbf{r})\right]
\end{aligned}
$$

with $\mathbf{E}(\mathbf{r})$ denoting now a real quantity. Here, it should be emphasized that

$$
\operatorname{Re}\left[\mathbf{d}(\omega) e^{i \omega t}\right]=\operatorname{Re}\left[\mathbf{d}^{*}(\omega) e^{-i \omega t}\right] .
$$

This mathematical remark is crucial for the later discussion about causality. Such "reset" enables now the introduction of a complex description in agreement with the electromagnetic convention (as used for the Larmor intensity in [1], but here with negative exponential):

$$
\begin{gathered}
\langle\mathbf{d}\rangle_{V}(t)=\operatorname{Re}\left[\overleftrightarrow{\alpha}_{M}(\omega) \mathbf{E}(\mathbf{r}) e^{-i \omega t}\right], \\
\overleftrightarrow{\alpha}_{M}(\omega)=\overleftrightarrow{\alpha}_{R}(-\omega)+\overleftrightarrow{\alpha}_{R}(\omega) .
\end{gathered}
$$

The complex polarizability $\alpha_{M}$ will be referred to as the monochromatic polarizability. Implicitly it also implies that in spite of the fact that only a single exponential has been used in the description, the amplitude of the real cosine-type wave can still be used. So, $\alpha_{M}$ can now explicitly be written as

$$
\begin{aligned}
\overleftrightarrow{\alpha}_{M}(\omega)= & \frac{q^{2}}{\hbar}\left(\frac{\omega_{l k}}{\omega}\right) \\
& \times\left[\frac{T_{r}(\omega)+i T_{\mathrm{i}}(\omega)}{\left[\left(\omega_{l k}-\omega\right)^{2}+\gamma^{2}(-\omega)\right]\left[\left(\omega_{l k}+\omega\right)^{2}+\gamma^{2}(\omega)\right]}\right] \\
\times & \times\langle k|\mathbf{r}| l\rangle_{V}\langle l|\mathbf{r}| k\rangle_{V}^{T}, \\
T_{r}(\omega)= & 2 \omega\left(\omega_{l k}^{2}-\omega^{2}\right)-\omega_{l k}\left[\gamma^{2}(-\omega)-\gamma^{2}(\omega)\right] \\
& -\omega\left[\gamma^{2}(-\omega)+\gamma^{2}(\omega)\right],
\end{aligned}
$$

$$
\begin{aligned}
T_{\mathrm{i}}(\omega)= & {\left[\omega_{l k}^{2}+\omega^{2}+\gamma(-\omega) \gamma(\omega)\right][\gamma(-\omega)+\gamma(\omega)] } \\
& +2 \omega \omega_{l k}[\gamma(-\omega)-\gamma(\omega)] .
\end{aligned}
$$

This expression for the monochromatic polarizability will be the final one to analyze the influence of the frequency dependence of the damping $\gamma(\omega)$ upon the electromagnetic response.

\section{NUMERICAL EXAMPLES}

On the basis of some numerical examples, the consequences of a particular choice for $\gamma(\omega)$ will be investigated. The frequency dependence for the damping will be like $\gamma(-\omega)$, since the quantum mechanics of the problem filters out the negative frequency. All polarizabilities introduced in the last section, will be written generically as

$$
\overleftrightarrow{\alpha}(\omega)=\frac{q^{2}}{\hbar}\langle k|\mathbf{r}| l\rangle_{V}\langle l|\mathbf{r}| k\rangle_{V}^{T} f(\omega)
$$

with the differences between the polarizabilities only arising from the frequency dependent function $f(\omega)$. First, monochromatic frequency dependent functions $f(\omega)$ for commonly used types of damping $\gamma(\omega)$ will be considered. The $f(\omega)$ assumes for defined parity $\gamma^{2}(-\omega)=\gamma^{2}(\omega)$ the form

$$
\begin{gathered}
f(\omega)=\left(\frac{\omega_{l k}}{\omega}\right)\left[\frac{T_{r}(\omega)+i T_{i}(\omega)}{\left[\omega_{l k}^{2}-\omega^{2}-\gamma^{2}(-\omega)\right]^{2}+4 \omega_{l k}^{2} \gamma^{2}(-\omega)}\right], \\
T_{r}(\omega)=2 \omega\left[\omega_{l k}^{2}-\omega^{2}-\gamma^{2}(-\omega)\right] .
\end{gathered}
$$

The defined parity reduces only the real part $T_{r}(\omega)$. A change of parity, on the other hand, affects only the imaginary part $T_{i}(\omega)$ and leaves the following choices:

$$
\begin{gathered}
T_{E, i}(\omega)=2 \gamma(-\omega)\left[\omega_{l k}^{2}+\omega^{2}+\gamma^{2}(-\omega)\right], \\
T_{O, i}(\omega)=4 \omega \omega_{l k} \gamma(-\omega),
\end{gathered}
$$

with the subscript $E$ referring to the even and the subscript $O$ to the odd parity for $\gamma(\omega)$. Notice that for both parities $T_{i}(\omega)$ is even. Apart from the even/odd parities, covering the body of the present sign discussion, there is also a proposition [16] to use an asymmetric choice $f_{A}(\omega)$, putting $\gamma(\omega)=0$ for $\omega$ $>0$. For completeness this option will be investigated as well:

$$
f_{A}(\omega)=\left(\frac{\omega_{l k}}{\omega}\right)\left[\frac{2 \omega\left(\omega_{l k}-\omega\right)-\gamma^{2}(-\omega)+i\left(\omega_{l k}+\omega\right) \gamma(-\omega)}{\left(\omega_{l k}+\omega\right)\left[\left(\omega_{l k}-\omega\right)^{2}+\gamma^{2}(-\omega)\right]}\right],
$$

with the subscript $A$ referring to this asymmetric choice. All these monochromatic options should be compared to the resonant type, denoted by the subscript $R$ :

$$
f_{R}(\omega)=\left(\frac{\omega_{l k}}{\omega}\right)\left[\frac{\omega_{l k}-\omega+i \gamma(-\omega)}{\left(\omega_{l k}-\omega\right)^{2}+\gamma^{2}(-\omega)}\right],
$$

the reference situation, but with an a priori already limited range of applicability. 


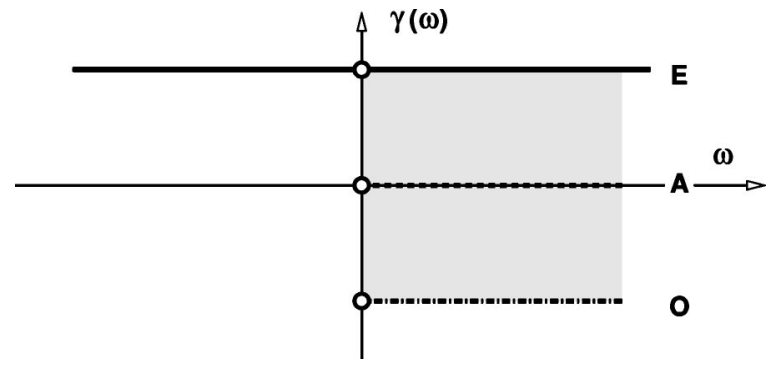

FIG. 1. "Constant" $\gamma(\omega)$ profile. Modulus $|\gamma|=0.1$ or 0.001 .

To determine which of the options proposed has to be preferred, the following four discriminating criteria will be used: The expression of $f(\omega)$ should be free from singular behavior; the expression should yield a positive absorbance; the symmetry with respect to $\omega$ should be correct; and causality should not be violated. The first round of investigation concerns the "constant" damping $\gamma(\omega)$ profiles shown in Fig. 1, which cover most of the current descriptions. In Figs. 2 and 3 are shown the functions $f_{R}(\omega)$ [Eq. (39)] for the resonant type, $f_{E, O}(\omega)$ [Eq. (37)] for the even and odd parity, and $f_{A}(\omega)$ [Eq. (38)] for the asymmetric choice. For $\omega_{l k}$ the value 1 will be taken and for the damping modulus $|\gamma|$ the values 0.1 and 0.001 . The latter value of 0.001 yields results closer to experiment. The former value of 0.1 is taken to highlight the details of and differences between the polarizabilities.

Near the resonance frequency $\omega=\omega_{l k}$ none of the cases is singular, provided that $\gamma\left(-\omega_{l k}\right) \neq 0$. A similar nonzero $\gamma(0)$ makes that all cases, except for the odd parity one, become singular in the real or imaginary part of $f(\omega)$ for $\omega=0$. In the odd parity case the imaginary part then becomes discontinuous. For the resonant type the singularity is even beyond repair, because only a single pole arises from the prefactor for $\omega=0$. As will be discussed later, in all other cases the singularity can be made to disappear if $\gamma(0)=0$ is assumed for a further smooth $\gamma(\omega)$. Near the resonance frequency there is hardly any difference between the separate cases. For $\gamma=0.1$, a pronounced difference occurs between the real parts of $f_{R}(\omega)$ and the other cases treated.

Because of the singularities occurring at $\omega=0$ for this "constant" $\gamma(\omega)$ profile, the near static region needs closer examination. The external electric field $\mathbf{E}_{X}$ applied to the volume element $V$, is given for any frequency $\omega$ by

$$
\mathbf{E}_{X}(\mathbf{r}, t)=\mathbf{E}_{X}(\mathbf{r}, \omega) \cos (\omega t) .
$$

Two mutually excluding descriptions are needed to derive this electric field from the electrodynamic potentials (18) as

$$
\begin{gathered}
\mathbf{E}_{X}(\mathbf{r})=-\nabla \Phi_{X}(\mathbf{r}), \\
\mathbf{E}_{X}(\mathbf{r}, \omega)=-\frac{\partial}{\partial t} \mathbf{A}_{X}(\mathbf{r}, \omega) \sin (\omega t) \\
=-\omega \mathbf{A}_{X}(\mathbf{r}, \omega) \cos (\omega t) .
\end{gathered}
$$

Since the electric field $\mathbf{E}_{X}(\mathbf{r}, \omega)$ is external, it can have any shape as a function of $\omega$. However, for a proper examination of the spectral behavior of the optical response a constant amplitude $\mathbf{E}_{X}(\mathbf{r}, \omega)$ should be taken. Therefore, the second description in Eq. (41) (also responsible for the prefactor and being undefined for $\omega=0$ ), should yield the same result in the limit $\omega \rightarrow 0$ as is produced by the first (static) description in Eq. (41). So this limit is the gradient of the scalar potential. It is incorrect to add it as a special static contribution in the static scalar potential gauge chosen. For very low frequencies and starting from $t=0$ there will be a time interval in which cos $\omega t$ will be virtually indistinguishable from 1 . The size of this interval will increase for decreasing $\omega$. It is unphysical to assume that in this interval the dipole strength $\langle\mathbf{d}\rangle_{V}(t)$ will be different for a slowly oscillating field of frequency $\omega$ and a static field of $t=0$ value. Therefore the response $\langle\mathbf{d}(\omega)\rangle$ has to be continuous in $\omega$ for $\omega=0$. As a result the polarizability $\alpha(\omega)$ and the frequency dependent function $f(\omega)$ have to be continuous for all frequencies $\omega$.

For the "constant" $\gamma(\omega)$ profile only the real parts of the even and odd frequency dependent functions have a proper static limit:

$$
\lim _{\omega \rightarrow 0} \operatorname{Re}\left[f_{E, O}(\omega)\right]=\frac{2 \omega_{l k}\left(\omega_{l k}^{2}-\gamma^{2}\right)}{\left(\omega_{l k}^{2}+\gamma^{2}\right)^{2}} .
$$

Yet for truly static fields $\mathbf{E}_{X}$ the total Hamiltonian has to be Hermitian, all (quasi)energies will be real and only $\gamma=0$ is possible. Therefore, there can be but one static value $f_{S}$ :

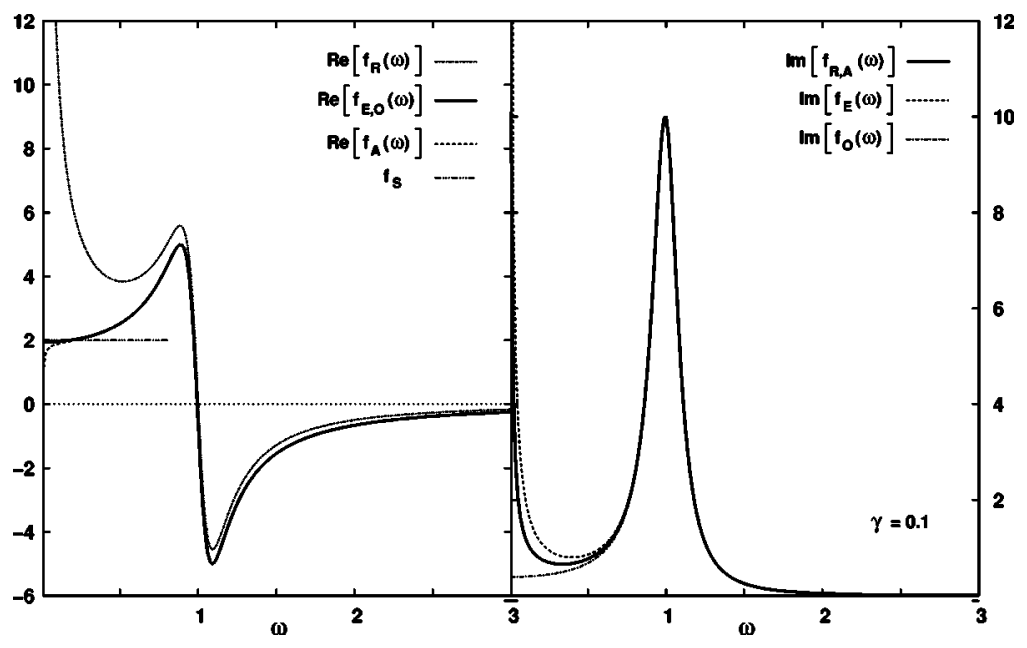

063807-6
FIG. 2. Constant frequency dependent damping: response functions $f(\omega)$ for resonant, asymmetric, odd, and even cases. Frequency $\omega$ and damping $\gamma$ in units of $\omega_{l k}$. Strong damping $\gamma$ $=0.1 f_{S}$ static limit. 


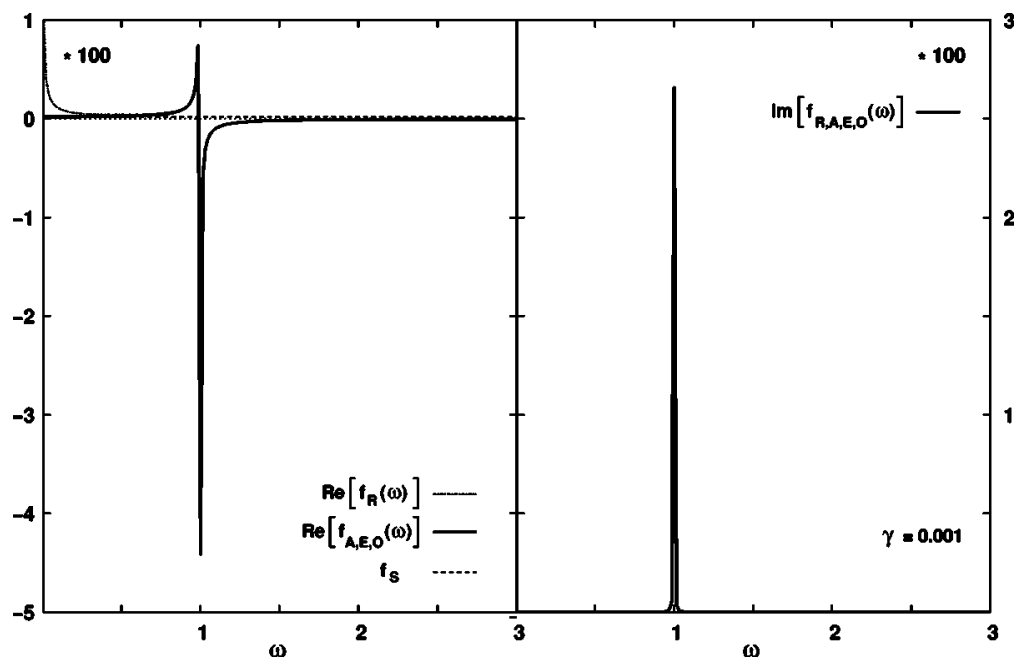

FIG. 3. Frequency dependent response functions $f(\omega)$ as in Fig. 2, but with weak damping $\gamma=0.001$.

$$
f_{S}=\frac{2}{\omega_{l k}}
$$

As a result, a discontinuity for this "constant" $\gamma(\omega)$ profile is left. Although the discontinuity is small (not visible, e.g., in Figs. 2 and 3), it remains unphysical.

For the investigation of the absorbance $\mathcal{A}$ of energy by the dipole from the field, Poynting's theorem yields that this absorbance is given by

$$
\mathcal{A}=\frac{\omega}{2} \operatorname{Im}\left(\mathbf{E}_{A}^{* T} \mathbf{p}\right)=\frac{\omega}{2} \mathbf{E}_{A}^{* T}\left(\operatorname{Im} \overleftrightarrow{\alpha}_{0}\right) \mathbf{E}_{A}
$$

For ordinary dissipative systems, as treated here, this quantity has to be positive in order not to violate conservation of energy. It suffices to investigate isotropic polarizabilities. Then it is immediately clear that the imaginary part of the polarizability has to be positive. All cases considered are in full agreement with this positive absorbance property of the electromagnetic energy conservation law.

Electromagnetism demands that the real part of the polarizability has to be even and the imaginary part odd, as a function of frequency. This arises directly from the electromagnetic requirement that

$$
\overleftrightarrow{\alpha}(-\omega)=\stackrel{\leftrightarrow}{\alpha}(\omega)
$$

for any polarizability $\alpha$. It is clear from Eq. (33) that this demand is obeyed by all monochromatic cases, regardless of the frequency dependence of $\gamma(\omega)$. Only the resonant type is not in agreement with this demand.

The last issue to be investigated concerns causality. To that end the polarizabilities, or rather the frequency dependent functions, have to obey the Kramers-Kronig (KK) transform. For that analysis it is better to use the direct precursor of the KK transform:

$$
f(\omega)=\frac{1}{i \pi} \mathcal{P} \int_{-\infty}^{\infty} d \omega^{\prime} \frac{f\left(\omega^{\prime}\right)}{\omega^{\prime}-\omega},
$$

where $\mathcal{P}$ stands for the principal value. Mathematically, the KK transform requires the frequency dependent function to be analytical in the upper part of the complex $\hat{\omega}$ plane. In practice this means that the frequency dependent function has to be free from poles in the upper half plane. First, the frequency dependent function $f_{R}(\hat{\omega})$,

$$
f_{R}(\hat{\omega})=\frac{1}{\hat{\omega}_{l k}-\hat{\omega}},
$$

will be analyzed. The prefactor will be suppressed, since it will turn out that its pole contributions can be ignored for the monochromatic cases. Equation (46) for $f(\omega)$ is correct only if the following contour integral, using for $f(\omega)$ the particular form of $f_{R}(\hat{\omega})$ given in Eq. (47):

$$
\begin{aligned}
\int_{\Gamma} d \hat{\omega}^{\prime} \frac{f_{R}\left(\hat{\omega}^{\prime}\right)}{\hat{\omega}^{\prime}-\omega} & =\left(\frac{1}{\hat{\omega}_{l k}-\omega}\right)\left[\int_{\Gamma} \frac{d \hat{\omega}^{\prime}}{\hat{\omega}^{\prime}-\omega}+\int_{\Gamma} \frac{d \hat{\omega}^{\prime}}{\hat{\omega}_{l k}-\hat{\omega}^{\prime}}\right] \\
& =f_{R}(\omega) \int_{\Gamma} \frac{d \hat{\omega}^{\prime}}{\hat{\omega}_{l k}-\hat{\omega}^{\prime}}
\end{aligned}
$$

becomes zero. Then, the function $f_{R}$ complies with the KK transforms. This happens only when $\hat{\omega}_{l k}$ has a negative imaginary part. Taking causality and fulfillment of the KK transform to be equivalent, $f_{R}(\hat{\omega})$ can be causal only if $\hat{\omega}_{l k}$ has a negative imaginary part.

Two classes of poles have to be distinguished in the frequency dependent functions belonging to the $\gamma(\omega)$ profiles of Fig. 1. First, there are the poles occurring at the origin, because of the prefactor. These poles are located on the integration contour $\Gamma$ and violate therefore the KK transform, because in practice they still contribute half of their residue. For this reason the resonant functions $f_{R}(\omega)$ are not causal. For the remaining options $f_{E}(\omega), f_{O}(\omega)$, and $f_{A}(\omega)$, there is a double pole for $\omega=0$. If $\gamma(\omega)$ is continuous and differentiable at $\omega=0$ and $\gamma(0)=0$, as for the "variable" $\gamma(\omega)$ to be treated next, the residues of these poles cancel. These poles will be ignored here.

The remaining poles are all related to the choice of $\hat{\omega}_{l k}$. The discussion will now focus upon the following two descriptions of the dipole strength and their corresponding frequency dependent functions: 

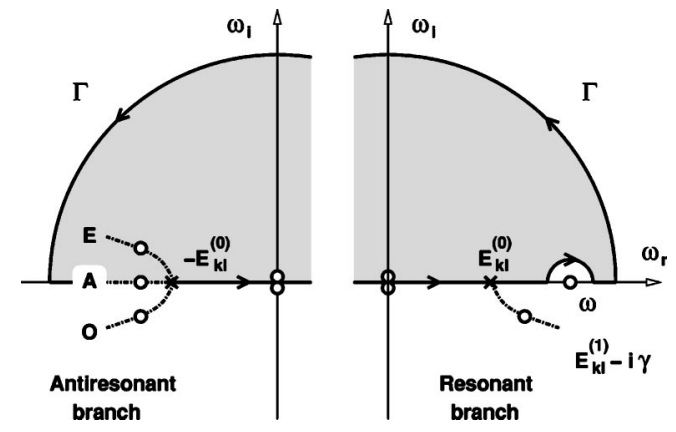

FIG. 4. Behavior frequency dependent functions in the complex plane. Resonant and antiresonant branches. Dash-dotted curves, "walking pole" trajectories; open circles, poles.

$$
\begin{gathered}
\langle\mathbf{d}\rangle(t)=\operatorname{Re}\left[\left(\overleftrightarrow{\alpha}_{R}(-\omega) e^{-i \omega t}+\overleftrightarrow{\alpha}_{R}(\omega) e^{i \omega t}\right) \mathbf{E}(\mathbf{r})\right], \\
\left\langle\mathbf{d}_{M}\right\rangle(t)=\operatorname{Re}\left[\left(\overleftrightarrow{\alpha}_{R}(-\omega)+\overleftrightarrow{\alpha}_{R}^{*}(\omega)\right) e^{-i \omega t} \mathbf{E}(\mathbf{r})\right] .
\end{gathered}
$$

The first line describes the response in its direct form given by Eq. (31), whereas the second, the monochromatic one, gives the response after the reset given by Eq. (32). For the direct description there are two different branches, the resonant branch with its pole in the right half plane and the antiresonant branch with its poles in the left half plane. This situation is depicted in Fig. 4. Since a cosine type of description has been chosen for the applied field, it suffices to consider only positive frequencies $\omega$, where a positive damping $\gamma(-\omega)$ will be taken for positive $\omega$. As can be seen from the expression for the resonant polarizability $\alpha_{R}(\omega)$, Eq. (30), there is only one relevant pole $\hat{\omega}$ for the resonant branch, at

$$
\hat{\omega}=\omega_{l k}-i \gamma(-\omega) .
$$

This pole is always in the lower half plane and this branch therefore will always be causal. For the antiresonant branch three options can be distinguished for the location of the pole: in the lower half plane (odd parity), on the real axis (asymmetric choice), and in the upper half plane (even parity). From these three cases only the odd parity one is causal, because it is the only case with the pole undoubtedly outside the contour $\Gamma$.

The monochromatic description behaves differently. Now there is a single frequency dependent function containing all of the poles discussed for the direct form, but with the antiresonant even and odd poles reversed (Fig. 5). This results straight from the reset enabled by Eq. (32), because the imaginary part of the dipole strength can actually be anything. By this reset the antiresonant poles become their complex conjugates. From the Cauchy-Riemann conditions it is clear that either the function itself or its complex conjugate can be analytic, but not both of them. This means that the monochromatic description will always be at odds with the direct one. Consider therefore the complex response, the quantity between square brackets in Eq. (49). The direct description follows straight from Schrödinger's equation and is causal only for odd parity. The monochromatic description is causal for even parity, but violates Schrödinger's equation. The odd parity wave function, however, increases exponen-

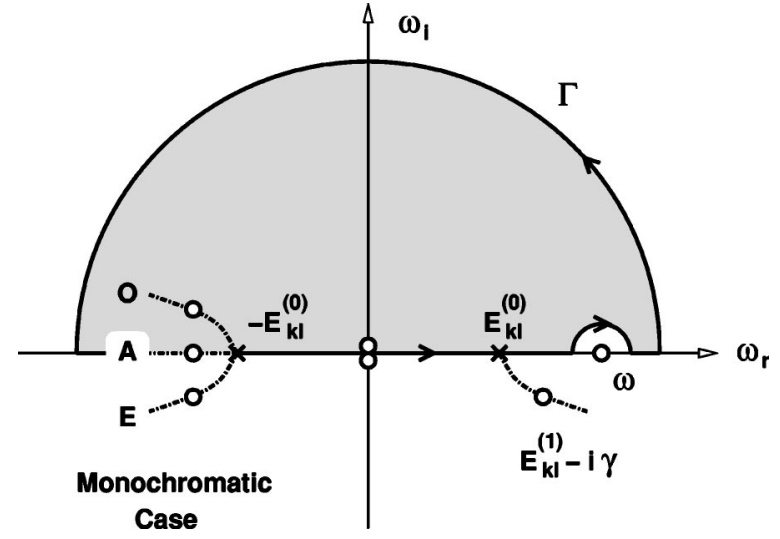

FIG. 5. Behavior frequency dependent functions in the complex plane. Monochromatic case. Dash-dotted curves, "walking pole" trajectories; open circles, poles.

tially with time, being unphysical. The even parity wave function has the correct damped behavior, but the necessary reset exploits explicitly that the imaginary part of the dipole strength has no physical meaning, according to Eq. (32). Yet the monochromatic description is not wrong and meets the demands from electromagnetism, requiring a single exponential description. The asymmetric choice will always violate the KK transform.

A remark of a different kind is more serious however. It has been assumed that the damping $\gamma(\omega)$ is a piecewise constant function of frequency. Inevitably it has still to be maintained that $\gamma(0)=0$, because it results from the constraints for $\hat{H}_{D}(\omega)$ and it cures the singularity problems for $\omega=0$ in all monochromatic cases. Therefore, $\gamma(\omega)$ really has to be a function of $\omega$. This means that the poles cannot have a fixed location in the complex plane, but can at best be described as "walking poles" in this picture (Figs. 4, 5). On the basis of full knowledge of $\gamma(\omega)$, or $\hat{H}_{D}(\omega)$, complex analysis can be done properly. This premise however, is beyond the scope of this paper.

In a next round of investigation, the consequences of a "variable" damping $\gamma(\omega)$ profile will be shown. The modeling of this $\gamma(\omega)$ profile (Fig. 6) will be as follows:

$$
\gamma_{E}(\omega)=\gamma\left(\frac{2 \omega_{l k}^{2} \omega^{2}}{\omega_{l k}^{4}+\omega^{4}}\right)
$$

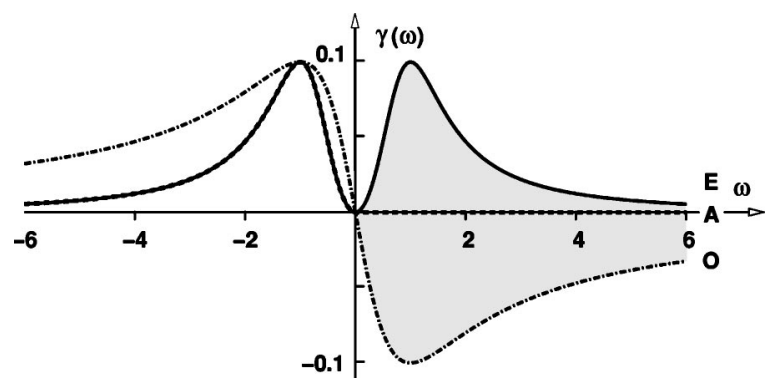

FIG. 6. "Variable" $\gamma(\omega)$ profile. Amplitude $\gamma=0.1$. 


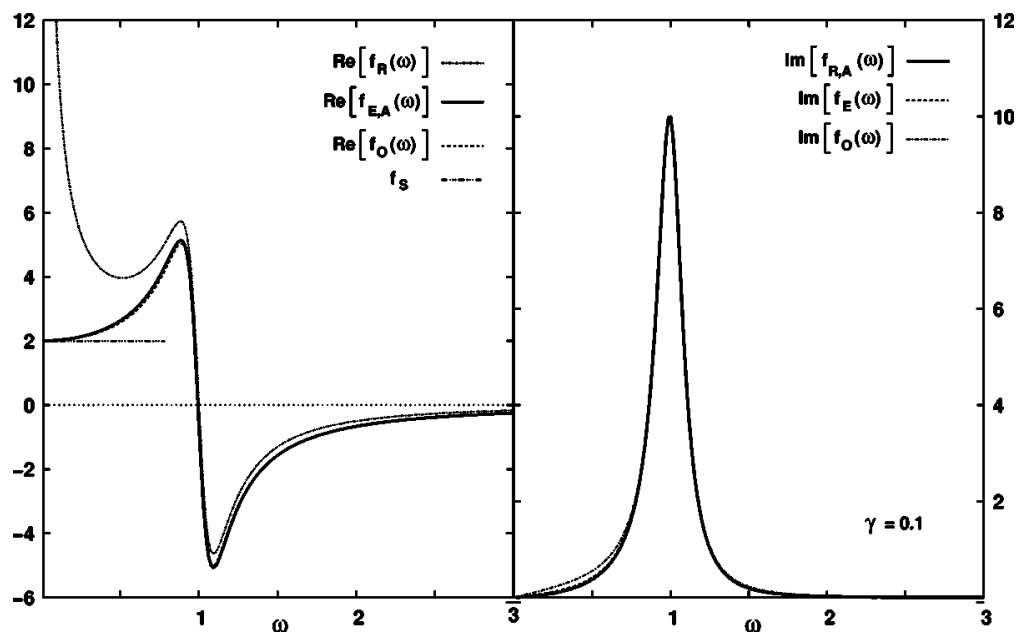

FIG. 7. Variable frequency dependent damping: response functions $f(\omega)$ as in Fig. 2 with $\gamma$ $=0.1 . \gamma(\omega)$ according to Eq. (51).

$$
\gamma_{O}(\omega)=-\gamma\left(\frac{2 \omega_{l k} \omega}{\omega_{l k}^{2}+\omega^{2}}\right)
$$

The asymmetric choice $\gamma_{A}(\omega)$ follows $\gamma_{E}(\omega)$ for negative frequencies and is 0 otherwise. Also for the resonant type $\gamma_{E}(\omega)$ is used. The sign definition for $\gamma(\omega)$ is as described by Eq. (1). In the modeling it has been used that at infinity there is no damping. This assumption is only based upon the classical argument, that a mass point will not follow very fast oscillations and becomes effectively static without energy transfer. The frequency dependent functions $f_{A, E, O, R}(\omega)$ belonging to this "variable" $\gamma(\omega)$ profile are shown in Fig. 7. The real part of $f_{R}(\omega)$ clearly deviates from all other cases. The real parts for these other cases are almost similar and the same holds for all imaginary parts, including resonant. The limiting behavior of the real and imaginary parts near $\omega=0$ correctly yields $f_{S}$, as given in Eq. (43), for all cases, apart from resonant. The results shown before in Fig. 2, for a "constant" $\gamma(\omega)$, represent traditional Lorentz line shapes. As compared to the Lorentzian, the distortion introduced by the variable $\gamma(\omega)(51)$ is also small.

In Table I the major conclusions of this paper have been summarized, in order to compare the different cases investigated. The labels $E, O, A, R$ in the first column are the frequency dependent function labels. The columns 2, 3, 4, 5

TABLE I. Performance of polarizability models using even $(E)$, odd $(O)$, asymmetric $(A)$, or resonant $(R)$ frequency dependence $f(\omega)$. Columns 2, 3, 4, and 5, no singular behavior for $\omega=0$; column 6, absorbance $\mathcal{A}>0$, Eq. (44) ; column 7, excited states $\phi_{l}^{(1)}$ damped; columns 8 and 9 , causality obeyed, $D$ direct, $M$ monochromatic, Eq. (49). Further, see text.

\begin{tabular}{cccccccccc}
\hline \hline & \multicolumn{3}{c}{ Constant $\gamma$} & \multicolumn{3}{c}{ Variable $\gamma$} & & & \multicolumn{3}{c}{ Causality } \\
& $\operatorname{Re}(\alpha)$ & $\operatorname{Im}(\alpha)$ & $\operatorname{Re}(\alpha)$ & $\operatorname{Im}(\alpha)$ & $\mathcal{A}>0$ & $\phi$ damped & $D$ & $M$ \\
\hline$E$ & + & - & + & + & + & + & - & + \\
$O$ & + & $\sim$ & + & + & + & - & + & - \\
$A$ & - & - & + & + & + & $\sim$ & $\sim$ & $\sim$ \\
$R$ & - & - & - & + & + & + & + & + \\
\hline \hline
\end{tabular}

concern the frequency dependent behavior of the real and imaginary parts of the polarizability $\alpha(\omega)$. For the two options used for the $\gamma(\omega)$ profile, constant and variable, the symbols,-+ indicate whether or not these polarizability components become singular for $\omega$ approaching 0 . The $\sim$ used four times in the table indicates that additional comment is required there, e.g., for the constant $\gamma$ profile, the imaginary part of the odd parity polarizability is not singular for $\omega=0$, but discontinuous. Columns 6 and 7 summarize absorption related features. From the electromagnetic point of view, the absorption is given by Eq. (44) and should be positive. All cases obey this demand (column 6). Column 7 refers to the time dependent behavior of the excited state $\phi_{l}^{(1)}$. If this state is damped for $t \rightarrow \infty$, it is shown in the table by $\mathrm{a}+$. The $\sim$ for the asymmetric choice in column 7 originates from the fact that the antiresonant part is related to a state with time independent modulus. The last two columns 8 and 9 summarize the behavior of the poles for the direct and monochromatic descriptions, according to Eq. (49). The + indicates that the antiresonant pole is outside the contour $\Gamma$ and the behavior has to be classified as causal. Since the antiresonant pole for the asymmetric choice is on the real axis, such classification cannot be given.

Regarding the table and requiring that $\gamma(0)=0$, so ignoring columns 2 and 3, the best solution is given by the even monochromatic one, in agreement with the original demands from quantum mechanics. Neither the damping of the wave function nor the issue of causality (columns 7, 8, and 9) can be addressed directly in an experiment. When also these columns 7, 8, and 9 are left out of consideration, it is not possible to choose between even and odd parity.

Finally, as in the original KH paper, the pair results can be extended to treat approximately systems with an arbitrary number of levels. To that end the contributions of all pairs $l k$, representing transitions from any occupied state $k$ to any unoccupied state $l$, have to be added to produce the sumover-states expression. For the induction rule given by

$$
\langle\mathbf{d}\rangle_{V}(t)=\operatorname{Re}\left[\mathbf{d}(\omega) e^{-i \omega t}\right]=\operatorname{Re}\left[\stackrel{\leftrightarrow}{\alpha}_{G}(\omega) \mathbf{E}_{X}(\mathbf{r}) e^{-i \omega t}\right]
$$

the definition for the total polarizability $\alpha_{G}$, if the damping $\gamma(\omega)$ is chosen to have even parity, becomes then 


$$
\begin{aligned}
\overleftrightarrow{\alpha}_{G}(\omega)= & \frac{2 q^{2}}{\hbar} \sum_{k l}\left(\frac{\omega_{l k}}{\omega}\right) \\
& \times\left[\frac{\omega\left(\omega_{l k}^{2}-\omega^{2}-\gamma_{l k}^{2}\right)+i \gamma_{l k}\left(\omega_{l k}^{2}+\omega^{2}+\gamma_{l k}^{2}\right)}{\left(\omega_{l k}^{2}-\omega^{2}-\gamma_{l k}^{2}\right)^{2}+4 \gamma_{l k}^{2} \omega_{l k}^{2}}\right] \\
& \times\langle k|\mathbf{r}| l\rangle_{V}\langle l|\mathbf{r}| k\rangle_{V}^{T},
\end{aligned}
$$

where the indices $k l$ of the damping $\gamma$ have been restored. Different from before, we have defined $\mathbf{d}(\omega)$ for this final expression without minus sign, to be in agreement with existing definition schemes in optics. The near static behavior is, of course, as treated before, but one warning should be made. When measuring in the uv-visible range it is not difficult to find a low frequency "static" response. Yet this response is seldom exactly the same as the result from a static measurement. At least partly this discrepancy has to be assigned to the contribution of infrared vibrational levels, when the volume $V$ is not occupied by a simple atom.

Some comment should be given as to the tensorial aspects of the expressions for the polarizability. If some rotation is applied to the system by means of a rotation matrix $\mathcal{R}$, the result

$$
\overleftrightarrow{\alpha}^{\prime}=\mathcal{R} \overleftrightarrow{\alpha} \mathcal{R}^{T}=\frac{q^{2}}{\hbar}\left[\mathcal{R}\langle l|\mathbf{r}| k\rangle_{V} \mathcal{R}^{T}\right]\left[\mathcal{R}\langle l|\mathbf{r}| k\rangle_{V} \mathcal{R}^{T}\right]^{T} f(\omega)
$$

simply states that the rotated polarizability is the direct product of the rotated matrix elements $\langle k|\mathbf{r}| l\rangle_{V}$. As a result, always a coordinate system can be chosen having one of the axes coinciding with the matrix element. Then the polarizability tensor has only one nonzero element somewhere on the diagonal. So a two-level description inevitably always ends up in a highly anisotropic polarizability. Most atoms, nevertheless, respond isotropically for symmetry reasons. The same symmetry however, gives rise also to degenerate levels. When this degeneracy is taken into account, isotropic behavior is again retained, using the summation in (53). In the original $\mathrm{KH}$ paper only isotropic cases were studied. It is, however, easy to go beyond this restriction (see also, e.g., $[2,4])$ and to obtain the full tensor as well.

\section{CONCLUSIONS}

The polarizability of a small arbitrary volume element $V$ has been derived, using the expectation value of the dipole strength, as in the original Kramers-Heisenberg paper. Different from $\mathrm{KH}$, electromagnetic potentials have been used for the field description and a separate dynamic dissipative part for the effective single electron Hamiltonian. The latter gives rise to a frequency dependent damping factor that is zero in the static case, as is generally accepted now in most phenomenological descriptions. This semiclassical nonrelativistic derivation takes into account only damping by the material system in the weak field limit. The use of potentials results in a prefactor reciprocal in $\omega$, as discussed by Lamb [15]. This prefactor arises from the use of Ehrenfest's theorem, but has hardly any visible consequences for the monochromatic cases studied here. The potential description requires a gauge transform to be chosen. In order to preserve the static scalar potential commonly used in quantum mechanics, Poisson's law has to be used to replace the Coulomb gauge. This is possible only when the electromagnetic fields used in the harmonic perturbation are external to the volume $V$.

It has been shown that a reset of the antiresonant branch of the polarizability is necessary to obtain results of the type commonly used in electromagnetism and referred to as monochromatic. This reset is mathematically correct, but does not follow naturally from the quantum mechanics of the problem. Explicitly, two types of frequency dependent damping profile, constant and variable, have been investigated. Both profile types have been studied as even or odd symmetric and as asymmetric monochromatic functions of frequency. Results have been compared to the generally accepted resonant results. The treatment of the frequency dependence of the damping $\gamma(\omega)$ is also relevant for the present discussion in the literature about the sign of the damping for the antiresonant term [4-6]. Absence of near static smooth behavior of the damping, as shown by the constant damping cases, unavoidably ends up in unphysical behavior for that frequency range. Regarding only the monochromatic polarizability for the variable damping profile, there is no clear preference for any kind of damping symmetry with respect to frequency. On the basis of purely theoretical arguments, such as causality and energy conservation, preference can be given to the even type of variable frequency dependent damping. Such preference is in agreement with the a priori assumptions concerning the behavior of the wave functions. The odd parity is preferred in treatments where the radiation field is part of the quantum mechanical description. Radiation losses have not been taken into account and have to be added separately when necessary, e.g., classically by means of a Lorentz radiation damping term.

\section{ACKNOWLEDGMENT}

The author wishes to thank Ir. H. H. J. M. Niederer for his careful reading of the manuscript and interesting discussions.
[1] H. A. Kramers and W. Heisenberg, Z. Phys. 31, 681 (1925).

[2] P. A. M. Dirac, Proc. R. Soc. London, Ser. A 114, 710 (1927).

[3] V. Weiskopf and E. Wigner, Z. Phys. 63, 54 (1930); 65, 18 (1930).
[4] A. D. Buckingham and P. Fischer, Phys. Rev. A 61, 035801 (2000).

[5] D. L. Andrews, L. C. Dávila Romero, and G. E. Stedman, Phys. Rev. A 67, 055801 (2003).

[6] P. W. Milonni and R. W. Boyd, Phys. Rev. A 69, 023814 
(2004).

[7] M. Rohlfing and S. G. Louie, Phys. Rev. B 62, 4927 (2000).

[8] W. Heitler, The Quantum Theory of Radiation (Clarendon, Oxford, 1960).

[9] W. P. Healy and R. G. Woolley, J. Phys. B 11, 1131 (1978).

[10] D. J. Boers and C. M. J. Wijers, Europhys. Lett. 60, 606 (2002).

[11] L. Rosenfeld, Theory of Electrons (Dover, New York, 1965).
[12] M. O. Scully and M. S. Zubairy, Quantum Optics (Cambridge University Press, Cambridge, U.K., 1997).

[13] C. Cohen-Tannoudji et al., Quantum Mechanics (Wiley, New York, 1997).

[14] C. M. J. Wijers and P. L. de Boeij, J. Chem. Phys. 116, 328 (2002).

[15] W. E. Lamb, Jr., Phys. Rev. 85, 259 (1952).

[16] P. P. Shorygin, Sov. Phys. Usp. 16, 99 (1973). 